\title{
ON THE EFFICIENCY OF A CONICAL UNDER-PLATFORM DAMPER FOR TURBINES
}

\author{
E. Denimal \\ Department of Mechanical Engineering \\ Imperial College London \\ London SW7 2AZ, UK \\ Email: e.denimal@imperial.ac.uk \\ L. Salles \\ Department of Mechanical Engineering \\ Imperial College London \\ London SW7 2AZ, UK \\ Email: I.salles@imperial.ac.uk
}

\author{
C. Wong \\ Rolls-Royce plc \\ Derby DE2 48J, UK \\ Email: Chian.Wong@Rolls-Royce.com
}

\author{
L. Pesaresi \\ Department of Mechanical Engineering \\ Imperial College London \\ London SW7 2AZ, UK \\ Email: I.pesaresi@imperial.ac.uk
}

\section{ABSTRACT}

Underplatform Dampers are commonly used in aircraft engines to limit the risk of High-Cycle Fatigue of turbine blades. The latter is located in a groove between two consecutive blades. The dry friction contact interface between the damper and the blades dissipates energy and so reduces the vibration amplitudes. Two common geometries of dampers are used nowadays, namely wedge and cylindrical dampers, but their efficiency is limited when the blades have an in-phase motion (or a motion close to it), since the damper tends to have a pure rolling motion. The objective of the present study is to analyse a new damper geometry, based on a conical shape, which prevents from this pure rolling motion of the damper and ensures a high kinematic slip. The objective of this study is to demonstrate the damping efficiency of this geometry. Hence, in a first part, the kinematic slip is approximated with analytical considerations. Then, a nonlinear dynamic analysis is performed, and the damping efficiency of this new geometry is compared to the wedge and the cylindrical geometries. The results demonstrate that the conical damper has a high damping capacity and is more efficient and more robust than the two others.

\section{INTRODUCTION}

Industrial requirements in the aerospace industry for gas turbine engines are becoming more stringent in terms of mass and efficiency, so that many components of such structures have reached their structural limits [1]. This is particularly true for turbines blades which are highly loaded, are subjected to high thermal, centrifugal stresses and vibrational stress [2]. The latter can lead to High Cycle Fatigue (HCF) and so to the failure of the blades [3]. Since the aero-engines have a large operating speed range and the modal density of bladed disk is high, it is impossible to avoid all critical resonances in the system. Hence, reducing the vibrations levels at these resonances is crucial, and solutions based on friction damping have been the most widely used over the years [4-6].

Dry friction can be introduced in different locations to limit the vibration levels, such as at the shrouds, the roots and blade tips. However, the most effective solution relies on the use of Underplatform Dampers (UPD) [6,7]. They consist in a metallic device placed in the groove under the platforms of two adjacent blades. The centrifugal loading keeps them in place during operation. If the blades vibrate, then the relative displacement between the platforms and the damper creates friction at the contact interface and so, energy is dissipated, and the system is damped $[8,9]$.

The identification of the most efficient UPD geometry is still an active research topic. Nevertheless, two main geometries have largely been studied and employed until now. The first shape is based on a wedge geometry [10-15] and the second shape is based on a cylindrical geometry $[12,16,17]$. Some studies 
present a combination of the two shapes [18-20] and how to optimise it [21].

The wedge geometry is the shape that is the most widely used over the years. It is easy to manufacture, install and replace, and it seals the platforms. However, with this shape, the prediction of the contact locations and conditions is difficult, which makes the modelling complex and the behaviour of the structure difficult to predict $[15,22]$. Moreover, each damper is loaded in a different way, which complicates even more the global dynamic of the system. The interesting feature of a cylindrical geometry is that the contact pressure is controllable and so the global behaviour of the structure is more predictable. However, both shapes have the same limitation. Indeed, when the blades have an in-phase motion, then the damper tends to have a pure rolling motion and no more frictional dissipation occurs. The dissipated energy is null or low, and the damping efficiency is limited.

The objective of the present study is to present a new damper geometry based on a conical shape. This geometry makes it impossible for the damper to have a pure rolling motion when the blades have an in-phase motion. In fact, to maintain contact with the two platforms, sliding is required so friction occurs. This friction is directly related to the kinematic motion of the damper created by the in-phase motion of the platform. Hence, the energy dissipated by friction is expected to be high as well as the damping efficiency.

The paper is organized as follows. In a first part, an approximation of the expected kinematic slip of this new damper is determined. It is based on an analytical analysis with a few assumptions. It gives quickly an approximation of the efficiency of the damper with regards to its geometrical characteristics. Then, for more accurate results, a dynamic analysis based on the Multi Harmonic Balance Method (HBM) is done, where the wedge, cylindrical and conical dampers are compared. The nonlinear receptances as well as the energy dissipated at the contact interface are considered to compare the efficiencies of the different dampers.

\section{NEW GEOMETRY PRESENTATION AND ANALYTIC PERFORMANCES}

Bladed disks are composed of a disc on which blades are fixed, and UPD are located under the platforms of adjacent blades. In this study, the focus is put on a system composed of two blades and one damper. If one considers the first bending mode of the blades, two extreme cases exist: an in-phase motion of the two blades and an out-of-phase motion. It is worth noticing that in reality, the blades have a mix of these two motions, but in the following, only these two "fundamental" conditions will be considered to study the behaviour of the damper. Considering these two cases, the kinematic of the platforms can be simplified, respectively, by a pure vertical or a pure horizontal motion of the platforms as illustrated Figure 1. For a horizontal

\section{Neutral position}

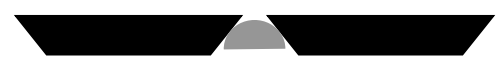

Platform horizontal motion (blades OOP)

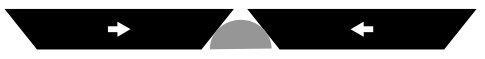

Platform vertical motion (blades IP)

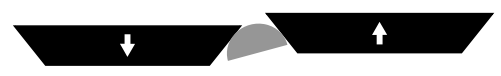

FIGURE 1: Illustration of movement of the platforms and the rolling motion

motion of the platforms, sliding occurs between the damper and the platforms, so energy is dissipated. However, in the case of a vertical motion, the damper tends to have a pure rolling motion and so the damper efficiency is very limited.

To cope with this issue, a different geometry is presented here. It is based on a conical shape as represented Figure 2. The damper is in blue and the planes defined by the platforms are in grey. The main feature of this geometry is that the rolling motion is not possible when a vertical motion of the platforms occurs. Indeed, to keep the contact with the two platforms and to keep its axis parallel to the platforms, the damper cannot have a pure rolling motion and so some sliding must occur. The latter creates friction and so energy is dissipated. This dissipation relies on the pure kinematic motion of the platforms and so a high efficiency of the damper is expected. The study is aimed to demonstrate and illustrate this basic idea. Moreover, this shape presents the advantage to have a robust description of the contact. Indeed the latter is reduced to a line which is easier to model, locate and gives a more controllable behaviour. This section is more specifically dedicated to analytical considerations.

\section{Axial Inclination of the Damper}

When the damper is positioned between the two platforms, its axial inclination is fully determined by the cone angle and the angle between the platforms. Moreover, the contact between the damper and each platform corresponds to a line which is also a generator of the cone. An illustration is given Figure 2. To determine the cone axial inclination, a few parameters need to be defined:

- $\gamma$ represents the axial inclination of the damper relative to the vertical. It is the angle between the plane formed by a section of the damper and the vertical,

- $\beta$ is the characteristic angle between the two platforms,

- $\alpha_{1}$ corresponds to the projection of the platform angle on the plane formed by the large horizontal section of the damper, 


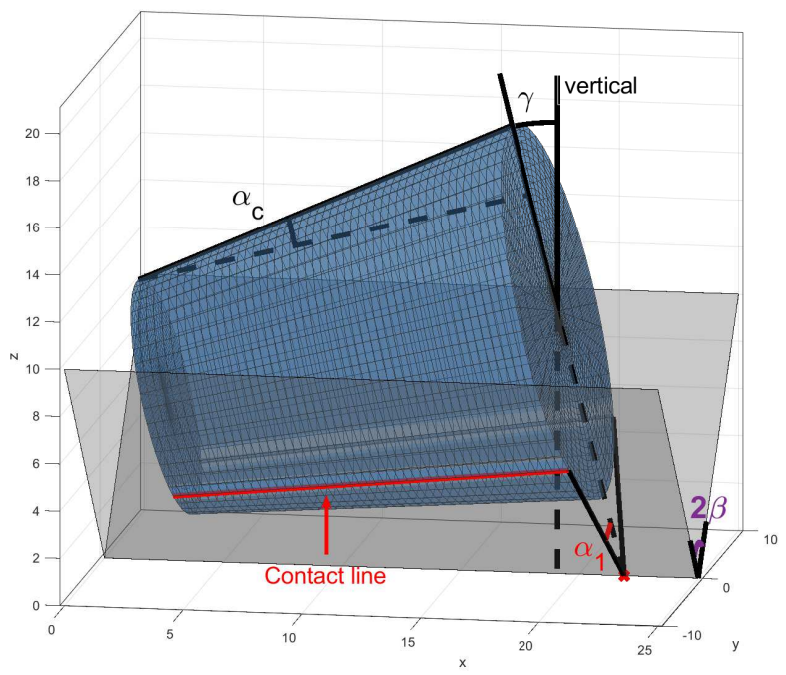

FIGURE 2: Illustration of the new damper geometry in contact with the platforms and the angles involved in the geometrical problem

- $\alpha_{c}$ is the semi angle of the cone.

The different angles are represented Figure 2.

From geometrical considerations, two relations can be obtained to relate the damper inclination $\gamma$ to the platform angle $\beta$ and the cone angle $\alpha_{c}$ :

$$
\left\{\begin{array}{l}
\tan \left(\alpha_{c}\right)=\tan (\gamma) \cdot \sin \left(\alpha_{1}\right) \\
\tan \left(\alpha_{1}\right)=\tan (\beta) \cdot \cos (\gamma)
\end{array}\right.
$$

From some manipulations it comes:

$$
\sin (\gamma) \tan (\beta)=\tan \left(\alpha_{c}\right) \sqrt{\left(1+(\cos (\gamma) \tan (\beta))^{2}\right)}
$$

which gives the following expression of the damper inclination $\gamma$ in function of the geometrical characteristic of the problem:

$$
\gamma\left(\alpha_{c}, \beta\right)=\arccos \left(\frac{\sqrt{\left(1-\tan ^{2}\left(\alpha_{c}\right) \cot ^{2}(\beta)\right)}}{\sqrt{\tan ^{2}\left(\alpha_{c}\right)+1}}\right)
$$

From Equation 3, the effect of the platform angle $\beta$ and of the cone angle $\alpha_{c}$ on the damper inclination can be determined. For example, Figure 3 gives the evolution of the damper inclination depending of the cone angle $\alpha_{c}$ for different platform angles $\beta$. As expected, when the damper is cylindrical, the inclination angle is null, and so the damper horizontal. On the opposite, when

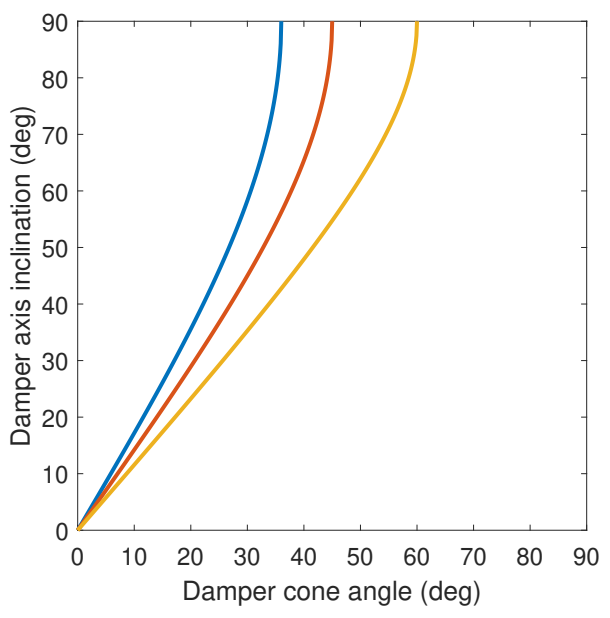

FIGURE 3: Damper inclination $\gamma$ versus the damper cone angle for different platform angles $\beta$ : $\beta_{1}=36^{\circ}$ (blue) $-\beta_{2}=45^{\circ}$ (orange) $-\beta_{3}=60^{\circ}$ (yellow)

the cone angle becomes equal to the platform angle, then the damper sits in a vertical position at 90 degrees with respect to the platforms.

\section{Kinematic Slip}

Once the damper inclination is determined, it is possible to get an approximation of the kinematic slip that will occur between the damper and the platforms. The latter is determined when a vertical motion of the platforms occurs, i.e. for the inphase family of modes of the blades. From this analytical approximation, it is possible to determine the influence of the different parameters on the expected damping efficiency. In order to estimate the kinematic slip, a few assumptions are necessary:

- the blades in-phase family of modes is simplified as a pure translational vertical motion between the platforms and the angle between the platforms cannot change,

- the damper and the platforms are always in contact,

- the damper and the platforms are perfectly rigid, and no elastic deformation is allowed,

- the middle of the damper is supposed to have a pure rolling motion.

The second and the last points are strong assumptions. The fullcontact assumption between the damper and the platforms comes from the idea that from the loading and the conical shape, the damper will always stay stuck to the platforms (no lift-off). The pure rolling motion of the middle section of the damper comes from the idea that the damper tends to have a symmetric motion to have similar displacements at each extremity. It is worth pointing out that these assumptions simplify significantly the problem and are only here to get a quick estimation of the kinematic slip 


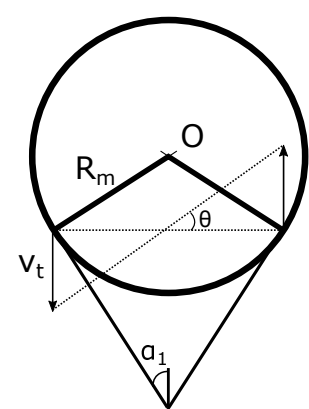

(a) Motion of the middle section of the damper

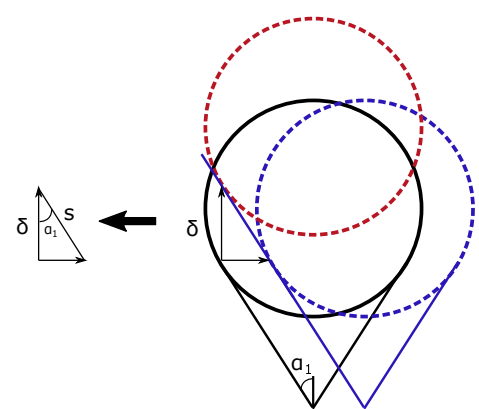

(b) Positions of the extreme section of the damper
FIGURE 4: Kinematic slip determination and position of the damper

in a preliminary process, rather than giving an accurate estimation of the damping potential for which simulations and tests are required.

Assuming a vertical motion of the platforms $v$, the vertical component in the plane of the middle section of the damper is $v_{t}=v \cdot \cos (\gamma)$, illustrated Figure 4a. So, if the middle section has a pure rolling motion, then a rotation $\theta$ appears with:

$$
\theta \approx \frac{v_{t}}{R_{m} \cos \left(\alpha_{1}\right)}
$$

where $R_{m}$ is the radius of the middle section. The smaller radius is denoted $R_{1}$ and the large radius $R_{2}$. The angle $\theta$ is assuming to be small, which is justified by the fact that the vibration amplitudes are extremely small compared to the geometrical dimensions. At the same time, each extreme section of the damper rotates of the same angle $\theta$. However, the distances travelled by the small and the large sections are different than the distance travelled by the middle section. The difference between the distance travelled by the middle section and the small (resp. large) section of the damper is equal to $\delta_{1}$ (resp. $\delta_{2}$ ):

$$
\delta_{1}=v_{t}-\frac{R_{1}}{R_{m}} v_{t} \quad \delta_{2}=v_{t}-\frac{R_{2}}{R_{m}} v_{t}
$$

Once the distance $\delta_{1}$ (resp. $\delta_{2}$ ) is travelled by the small (resp. large) section, the latter is not in contact with the other platform. In order to maintain contact with both platforms, it must slip the remaining distance denoted $s_{1}$ (resp. $s_{2}$ ). The different configurations of an extreme section of the damper are represented Figure $4 \mathrm{~b}$. The black configuration corresponds to the initial configuration, the red configuration to the configuration after travelling the distance $\delta$ and the blue configuration to the final configuration (i.e. $\delta$ and $s$ travelled). Hence, the kinematic slip corresponds to $s_{1}$ (resp. $s_{2}$ ) in Figure $4 \mathrm{~b}$ and is equal to:

$$
s_{1}=\frac{\left(1-\frac{R_{1}}{R_{m}}\right)}{\cos \left(\alpha_{1}\right)} v_{t} \quad s_{2}=\frac{\left(1-\frac{R_{2}}{R_{m}}\right)}{\cos \left(\alpha_{1}\right)} v_{t}
$$

A longitudinal kinematic slip $s_{l}$ also appears and is equal to $s_{l}=$ $v_{s} \cos \left(\alpha_{c}\right)$.

As an illustration, the absolute value of the kinematic slip at each position of the damper for different cone angles $\alpha_{c}$ is given Figure 5. The length of the damper is $L=40 \mathrm{~mm}$ and the platform angle is $\beta=45^{\circ}$. The kinematic slip is maximum at the extremities of damper and null at the centre as expected. Indeed, the main assumption if that the centre of the damper has a pure rolling motion, and so does not slip. The evolution is linearly dependent to the distance to the centre.

More generally, the maximum expected kinematic slip for different cone angles and for different configuration of the system can be determined. The influence of different parameters is illustrated Figure 6. In Figure 6a, the influence of the platform angle $\beta$ is studied. The evolution of the maximum of the kinematic slip is similar in each case. More precisely, the maximum normalized kinematic slip increases first when the cone angle increases, then it reaches a maximum before decreasing and becoming null when the cone angle is higher than the platform angle (i.e. when the damper has a vertical position). Hence, an increase of the platform angle tends to increase the maximum of the kinematic slip. Moreover, the maximum is reached for different values of the cone angle. Indeed, if $\beta=36^{\circ}$, the maximum is reached when $\alpha_{c}$ is around $55 \%$ of the platform angle, whereas if $\beta=60^{\circ}$ the maximum is reached around $44 \%$ of the platform angle. The influence of the radius of the large section $R_{1}$ and of the damper length $L$ are given Figures $6 \mathrm{~b}$ and $6 \mathrm{c}$, respectively. The evolution of the maximum of the kinematic slip is similar than in the case presented previously. However, an increase of the radius

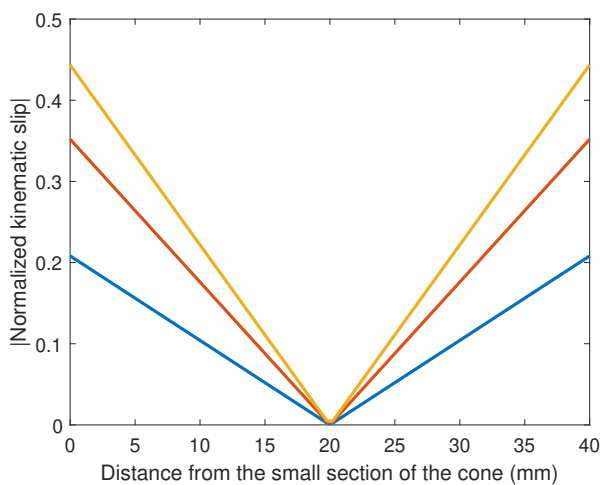

FIGURE 5: Evolution of the kinematic slip at each point of the damper for different cone angle $-\alpha_{c}=5^{\circ}$ (blue) $-\alpha_{c}=10^{\circ}$ (orange) $-\alpha_{c}=15^{\circ}$ (yellow) 
$R_{1}$ tends to decrease the maximum of expected slip: if $R_{1}=5 \mathrm{~mm}$ then the maximum is around 0.7 , whereas if $R_{1}=20 \mathrm{~mm}$ the maximum is around 0.3. Concerning the length of the damper, an increase of the latter tends to increase the maximum of the kinematic slip: if $L=40 \mathrm{~mm}$ then the maximum is around 0.4 , whereas if $L=60 \mathrm{~mm}$ then the maximum is around 0.6.

These different analytical considerations provide a coarse estimation of the expected kinematic slip and so of the damper efficiency. Moreover, they allow in a preliminary process to test and compare quickly the impact of some design parameters as the length of the damper, the cone angle or the radius of the sections.

\section{COMPARISON WITH OTHERS DAMPERS}

The analytical results given in the first part can be very useful in a first design process to identify the main characteristics of a damper. However, nonlinear dynamic simulations must be performed to get the real damping performances. The following part is devoted to the analysis of the performances of the conical damper to validate the analytical approach presented previously. The latter will be compared to a cylindrical and to a wedge damper.

\section{Model Presentation}

Blades Model The model used is the model of an underplatform test rig, used in previous works [15]. It has been designed to experimentally study the effect of UPDs on blade-like structure. The Finite Element Model (FEM) of the model is represented Figure $7 \mathrm{a}$ and is composed of two pseudo beam-like blades that are fixed on a common base which simulates a rigid disk. The dimensions of the model are chosen to mimic the behaviour of the dynamic response of a real HPT blade. The interested reader is invited to refer to [15] for more information. The mesh is composed of 54972 quadratic hexahedral elements and a stainless-steel material is used, i.e. the Young modulus is equal to $197 \mathrm{GPa}$ and the density is equal to $7800 \mathrm{~kg} / \mathrm{m}^{3}$. The first two modes of the structure correspond to the first bending mode of the blades, that can be in-phase or out-of-phase. The mode shapes are represented Figure $7 \mathrm{~b}$ and Figure $7 \mathrm{c}$ respectively.

Damper Models Three damper geometries are considered for this part of the study, they are represented Figure 7 . The first one is the wedge damper studied in [15] and represented Figure $7 \mathrm{~d}$. The second one is a conical damper of cone angle $10^{\circ}$ represented Figure 7e. Finally, the third one is a cylindrical damper with a radius equal to the mean radius of the conical damper, and is represented Figure 7f. The meshes are constructed so that the contact points are matching the blades mesh.

Contact Pressure In all cases, a uniform contact pressure

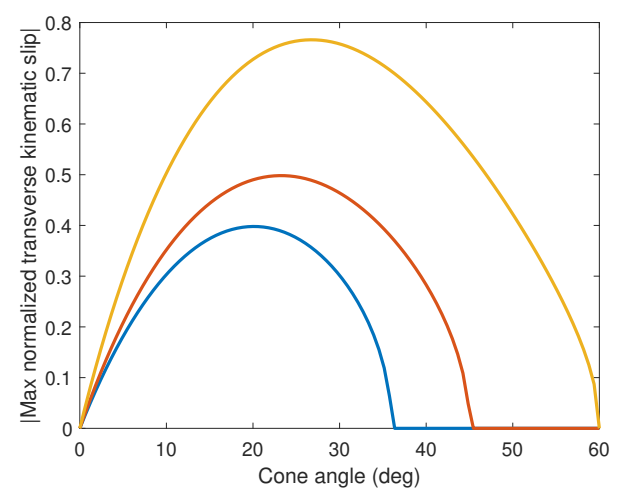

(a) Maximum kinematic slip versus the cone angle for different platform angles: $\beta_{1}=36^{\circ}$ (blue) $-\beta_{2}=45^{\circ}$ (orange) - $\beta_{3}=60^{\circ}$ (yellow)

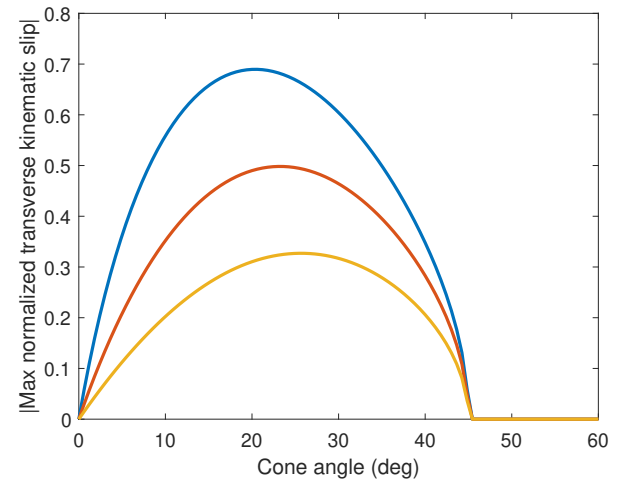

(b) Maximum kinematic slip for different radius $R_{1}$ of the cone: $R_{1}=5 \mathrm{~mm}$ (blue) $-R_{1}=10 \mathrm{~mm}$ (orange) $-R_{1}=$ $20 \mathrm{~mm}$ (yellow)

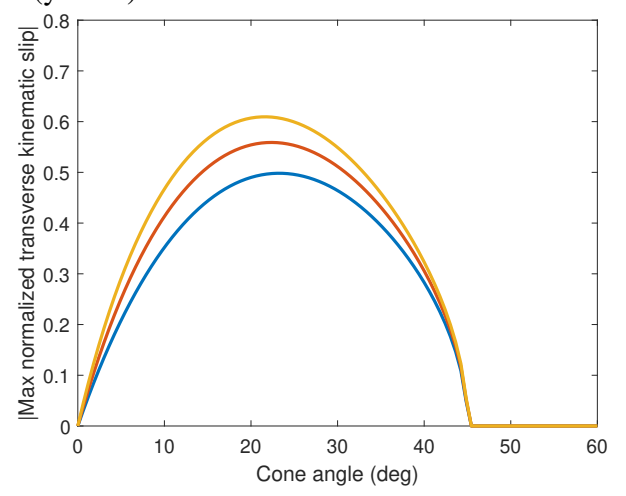

(c) Maximum kinematic slip for different length $L$ of the damper: $L=40 \mathrm{~mm}$ (blue) $-L=50 \mathrm{~mm}$ (orange) $-L=$ $60 \mathrm{~mm}$ (yellow)

FIGURE 6: Influence of different parameters on the maximum kinematic slip with regard to the cone angle $\alpha_{c}$

is assumed. For a wedge damper, the contact pressure due the centrifugal loading $C_{F}$ is modelled by a constant value [11] and 


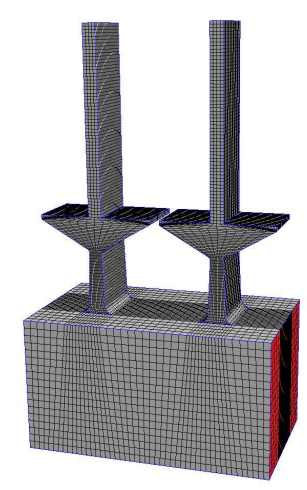

(a) Mesh of the platforms model

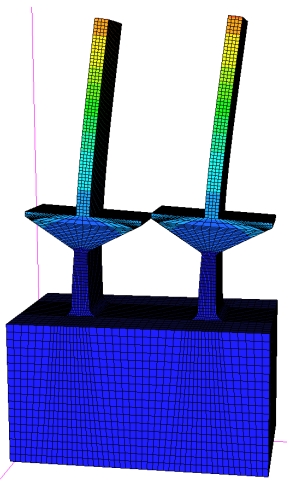

(b) In-phase mode $f_{\mathrm{IP}}=269.9 \mathrm{~Hz}$

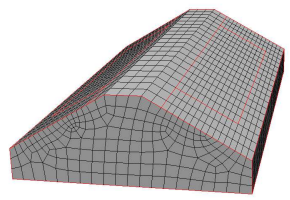

(d) Wedge

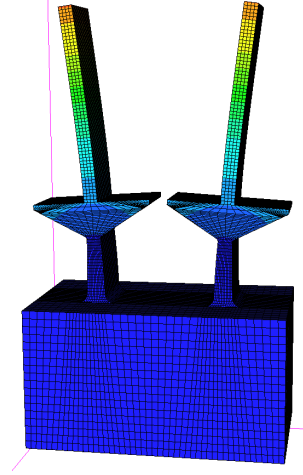

(c) Out-of-phase mode $f_{\text {OOP }}=269.8 \mathrm{~Hz}$

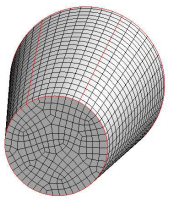

(e) Conical

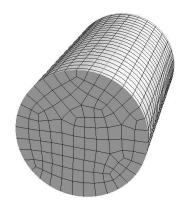

(f) Cylindrical
FIGURE 7: Finite element model of the blades model (a) and the first two modes of the structure (b,c) - FEM of the wedge (d), conical (e) and cylindrical (f) dampers

the initial pressure $\sigma_{0}$ on each side of the damper is equal to:

$$
\sigma_{0}=\frac{1}{2} \frac{C_{F}}{A(\cos \alpha+\mu \sin \alpha)}
$$

where $C_{F}$ is the radial centrifugal force, $A$ is the contact area on each side, $\mu$ is the friction coefficient and $\alpha$ is the damper angle. For the cylindrical and the conical damper, it is assumed that the total contact force remains the same. Since the contact between the damper and the platform is a line in these cases, the total contact force is divided by the number of points (the points are regularly positioned).
Contact Formulation A node-to-node contact formulation is used to solve the problem. Thus, the contact surface of the wedge damper is reduced to 146 points as in [15], and the contact line for both the cylindrical damper and the conical damper is composed of 23 points (i.e. 46 in total).

The dynamic contact behaviour is described at each contact node by the 3D friction contact shown in Figure 8. This contact model is composed of two decoupled Jenkins elements [4] allowing for 2D in plane displacements, and a third spring is added to allow normal load variations. Hence, each element is characterized by four parameters, namely the friction coefficient $\mu$, the initial pre-load $N_{0}$, the tangential stiffness $k_{t}$ and the normal stiffness $k_{n}$. The normal pre-load and the stiffnesses are normalized by unit of area, so each contact element has its own characteristics. The contact formulation is the same for the different dampers and allows four different states for each point, namely stuck, stick/slip, partial separation and full separation.

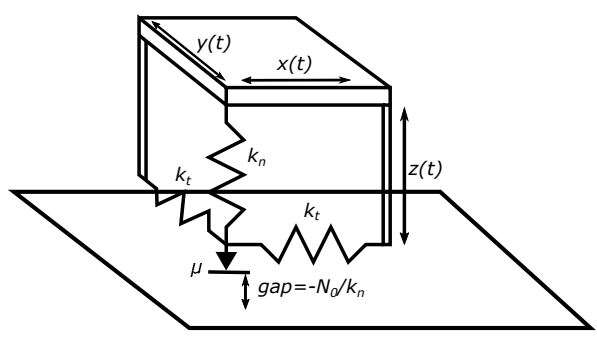

FIGURE 8: Contact model

\section{Harmonic Balance Method}

The numerical strategy used for the prediction of the vibrations levels of the system is based on the Multi-Harmonic Balance Method (MHBM) coupled with a model reduction technique included in the in-house code FORSE (FOrced Response SuitE), which is presented in details in [23-25]. The friction forces from the contact interface bring non-linearities in the system. The dynamic equation of the system can be written as:

$$
\mathbf{M X}+\mathbf{C} \dot{\mathbf{X}}+\mathbf{K X}+\mathbf{F}_{n l}(\dot{\mathbf{X}}, \mathbf{X})=\mathbf{P}(t)
$$

where $\mathbf{M}, \mathbf{C}$ and $\mathbf{K}$ are the mass, damping and stiffness matrices respectively. $\mathbf{X}$ is the vector of displacements and the dot corresponds to the derivative with respect to time. $\mathbf{P}$ is the vector of the external excitation and $\mathbf{F}_{n l}$ are the non-linear contact forces, that depend of the relative motion of nodes in contact at the interface.

The main idea of the MHBM consists in considering that the response of each DOF is periodic and so can be decomposed on 
a Fourier series which is truncated at the $n^{\text {th }}$ harmonic:

$$
\mathbf{q}(t)=\mathbf{Q}_{0}+\sum_{j=1}^{n} \mathbf{Q}_{j}^{c} \cos m_{j} \omega t+\mathbf{Q}_{j}^{s} \sin m_{j} \omega t
$$

where $\mathbf{Q}_{0}, \mathbf{Q}_{j}^{c}$ and $\mathbf{Q}_{j}^{s}$ are the Fourier coefficients. By injecting Eq. 9 into Eq. 8, and projecting on each harmonic, the system 8 can be approximated by:

$$
\mathbf{Z}(\omega) \mathbf{Q}+\tilde{\mathbf{F}}_{n l}(\mathbf{Q})-\tilde{\mathbf{P}}=\mathbf{0}
$$

where $\mathbf{Q}=\left\{\mathbf{Q}_{0}, \mathbf{Q}_{1}^{c}, \mathbf{Q}_{1}^{s}, \ldots, \mathbf{Q}_{n}^{c}, \mathbf{Q}_{n}^{s}\right\}$ is the vector of the Fourier coefficients, $\mathbf{Z}(\omega)$ is the dynamic stiffness matrix of the system, $\tilde{\mathbf{F}}_{n l}$ and $\tilde{\mathbf{P}}$ are the nonlinear forces and the external efforts in the Fourier basis respectively. The reduction of the model is based on a FRF matrix representation of the model. This FRF matrix is determined by calculating its exact value at some frequency points and adding a second term that describes its variation in a range of frequencies [25]. An Alternate Frequency/Time (AFT) [26, 27] algorithm is used for the determination of the nonlinear forces and the problem of Eq. 10 is solved with a Newton-Raphson algorithm. In order to compute a continuous response curve in the frequency domain, continuation is performed with regard to the pulsation $\omega$. Here, a secant predictor together with an arc-length corrector is used. In the following the harmonics 1, 2 and 3 are used for the simulations. They have been selected based on a convergence study. For the dampers and the blades, 12 modes are used for the simulation.

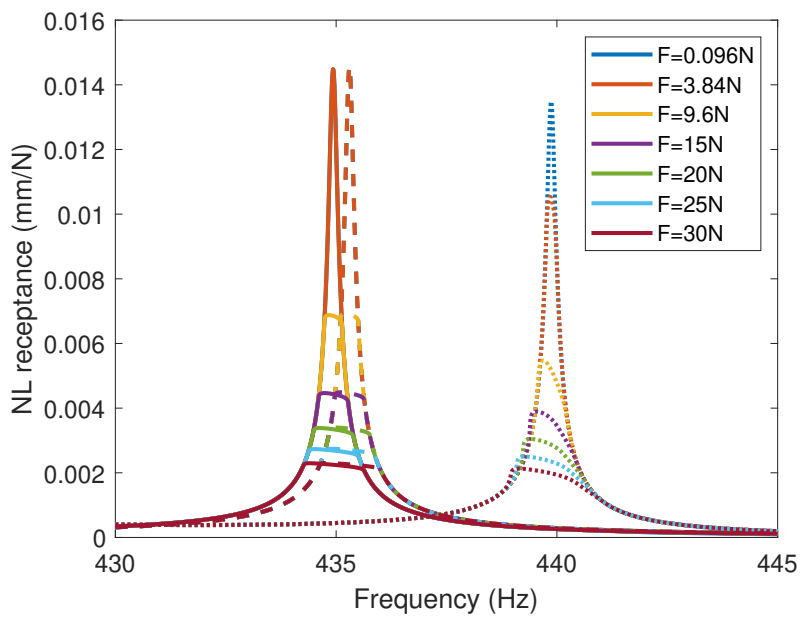

FIGURE 9: Nonlinear receptances for the out-of-phase mode: (-) conical damper - (- - -) cylindrical damper - (......) wedge damper

\section{Comparison of the Performances of the Different Dampers}

The nonlinear receptances of the three dampers are computed for different levels of excitation from $0.096 \mathrm{~N}$ to $30 \mathrm{~N}$. The receptances are given Figure 9 for the out-of-phase family case, and Figure 10 for the in-phase case.

The nonlinear receptances for the out-of-phase mode are given Figure 9 for the three cases. The curves with a solid line correspond to the conical damper receptances, the dashed ones to the cylindrical damper and the dotted ones to the wedge damper. The first observation is that compared to the cylindrical damper, the behaviour of the conical damper is almost identical with a small frequency shift of about $0.5 \mathrm{~Hz}$. In all cases, the three dampers have a similar behaviour and the damping efficiencies are comparable.

But considering the in-phase case, the behaviours of the three dampers are completely different (see Figure 10). Indeed, as a first observation, the resonance frequencies are spread from $309 \mathrm{~Hz}$ to $423 \mathrm{~Hz}$. As it can be seen Figure 10b, all the FRF are superimposed. It demonstrates that the damper has no effect on the dynamic of the blades, in other words the cylindrical damper has a pure rolling motion for this mode and the damping efficiency for this mode is very limited. Hence, the blades have a linear behaviour. Considering the wedge damper, it damps the response since the maximum of the receptances decreases when the excitation force increases but it also presents a strong softening behaviour and an important frequency shift (around $4 \mathrm{~Hz}$ of difference between the peak at $\mathrm{F}=0.096 \mathrm{~N}$ and the peak at $\mathrm{F}=30 \mathrm{~N}$ ). Finally, the conical damper presents a behaviour similar to the behaviour for the out-of-phase mode, i.e. a high damping efficiency without frequency shift.

The maximum of the absolute amplitude response is also given for the three dampers in Figure 11 for the in-phase case (Figure 11a) and the out-of-phase case (Figure 11b). For the outof-phase case, the three dampers have a similar behaviour. The amplitudes increase when the excitation increases and stabilize between $0.06 \mathrm{~mm}$ and $0.07 \mathrm{~mm}$ for high excitation levels. For the in-phase case, the three dampers have different behaviours. Indeed, as seen previously, the blades have a linear behaviour if the damper is the cylindrical one, so the absolute amplitude increases linearly with the excitation, which gives very high level of amplitude at high level of excitations. For the wedge damper (yellow curve in Figure 11a), the maximum of amplitude also increases with the level of excitation but more slowly and reached a maximum of $0.35 \mathrm{~mm}$ for $F=30 \mathrm{~N}$. But for the conical damper (blue curve), at very low level of excitations, the amplitude response increases but after $5 \mathrm{~N}$, it increases slightly from $7.8 \mathrm{E}-2 \mathrm{~mm}$ at $9.6 \mathrm{~N}$ to $8.7 \mathrm{E}-2 \mathrm{~mm}$ at $30 \mathrm{~N}$ of excitation amplitude, and almost does not depend of the excitation amplitude. This demonstrates the high damping capacity of the conical damper for the in-phase case. In fact, the damper is even more efficient than the wedge damper since there is a saturation effect. 


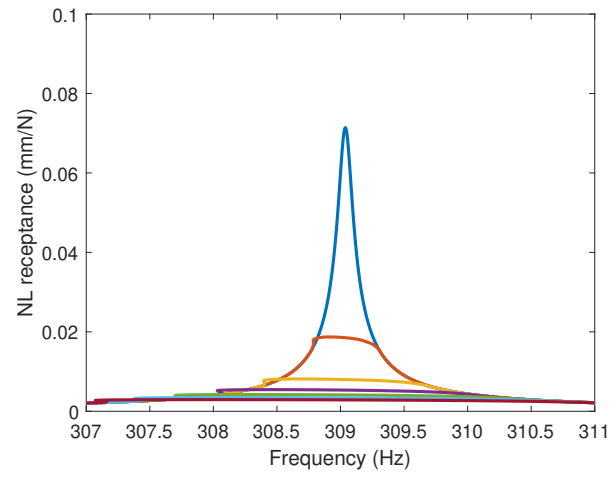

(a) Conical damper

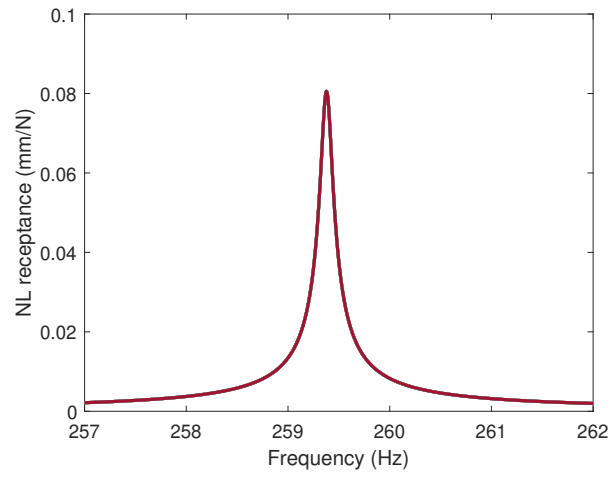

(b) Cylindrical damper

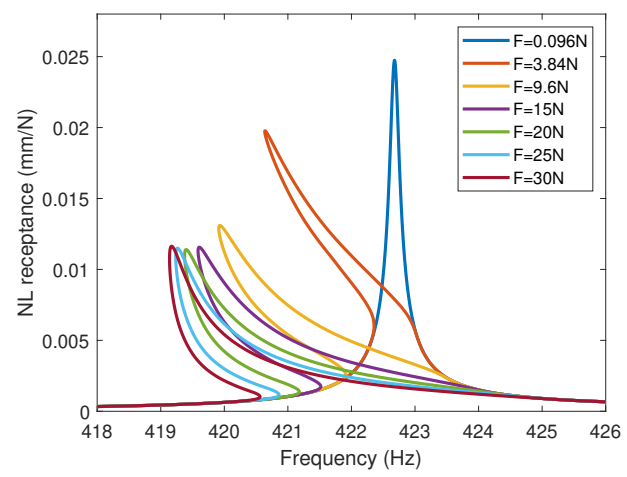

(c) Wedge damper

FIGURE 10: Nonlinear receptances for the in-phase mode: (left) conical damper - (middle) cylindrical damper - (right) wedge damper

To investigate more deeply the efficiency of the different dampers, the energy dissipated by friction for the different dampers and the different level of excitation are determined. They are given Table 1 for the out-of-phase case and Table 2 for

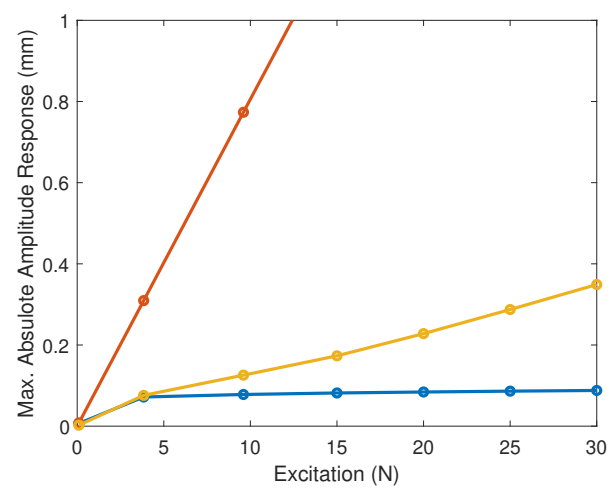

(a) In-phase mode

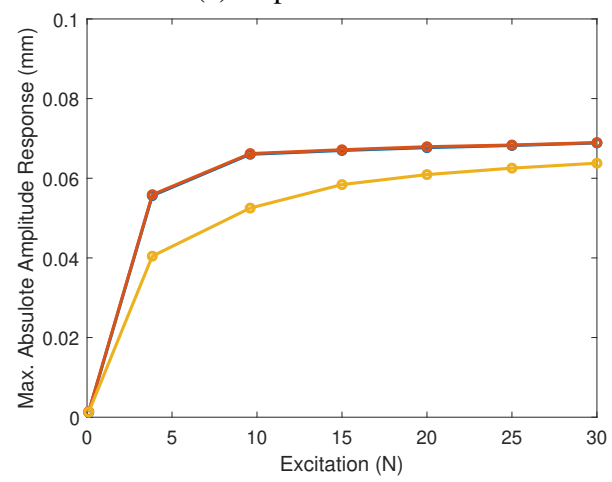

(b) Out-of-phase mode

FIGURE 11: Maximum of the amplitude response for different level of excitation for the different dampers: (blue) conical damper - (orange) cylindrical - (yellow) wedge damper the in-phase case. As expected, the level of energy dissipated by friction for the out-of-phase case is similar for the three dampers. Indeed, the quantity of dissipated energy is almost equal for the three dampers at each level of excitation (see Table 1). However, in the case of the in-phase mode family, the energy dissipated differs a lot from one damper to another. First, the cylindrical damper does not dissipate energy (see the low levels around e$18 \mathrm{~mJ}$ ), which confirms the pure rolling motion of the damper for this case. This analysis is confirmed by the evolution of the contact status of the contact points given Figure 12 for the different dampers for excitation levels equal to $9.6 \mathrm{~N}$ and $30 \mathrm{~N}$. For the cylindrical and the conical dampers, the points from 1 to 23 correspond to the left contact line, and the points 24 to 46 to the right. They are ordered so that the points 1 and 24 correspond to the large section and the points 23 and 46 to the small section. For the wedge damper, the points 1 to 73 (resp. 74 to 146) correspond to the left (resp. right) contact surface. Red points correspond to cases where stick/slip occurs at the contact and green points where separation occurs. As it can be seen, in the case of the cylindrical damper (top figures), friction occurs only for the out-of-phase case (i.e. at $409 \mathrm{~Hz}$ ). For the in-phase case, the contact points remain stuck and the damper has a pure rolling motion. Considering the conical damper, stick/slip occurs in both cases, for either the in-phase and the out-of-phase modes. Moreover, for the in-phase case, stick/slip appears at the extremities of the damper when the centre has a pure rolling motion (see the stuck points in the middle of each contact line). This confirms the assumption done in the analytical part. Moreover, no full contact separation is observed for the different amplitudes of excitation, justifying the full-contact assumption done in the analytical part. Since stick/slip occurs for the in-phase case, some energy is dissipated by friction and so the damper has, as expected, a damping effect on the system. Considering the wedge damper, the dynamic of the contact is much more complex for the in-phase case since almost all points experience contact sep- 
aration (see Figures 12e and 12f). This illustrates the rolling motion of the damper and explain the strong softening behaviour observed Figure 10c.

For more insight, the evolution of the repartition of the contact condition at the first resonance frequency for both the wedge and the conical dampers for different excitation amplitudes is given Figure 13. As it can be seen, for the conical damper (solid lines) the number of contact points in a stick/slip or partial contact state increases when the excitation amplitude increases. This evolution may explain why the maximum of the amplitude response remains almost constant in Figure 11a for the conical damper. Considering the wedge damper, when the excitation amplitude increases, all the points tend to experience contact separation. The energy dissipated by friction by the wedge damper

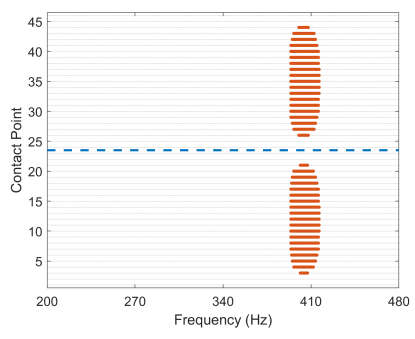

(a)

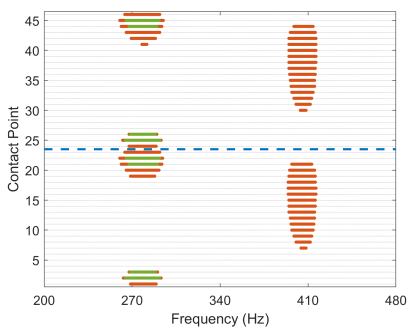

(c)

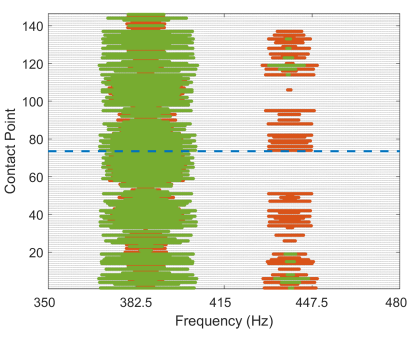

(e)

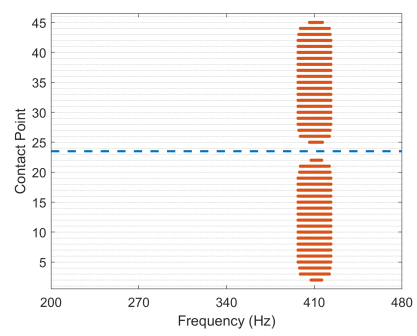

(b)

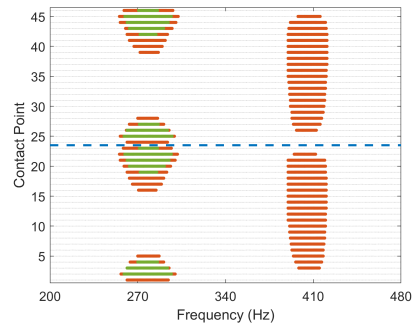

(d)

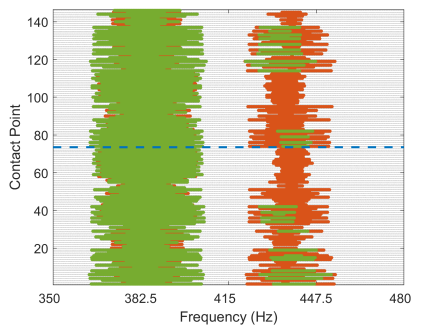

(f)
FIGURE 12: Evolution of the contacts status for the cylindrical (top), conical (middle) and wedge (bottom) dampers at different frequency levels for an excitation of $9.6 \mathrm{~N}$ (left) and $30 \mathrm{~N}$ (right):

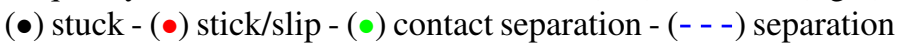
between contact points on the left side (bottom) and on the right side (top)

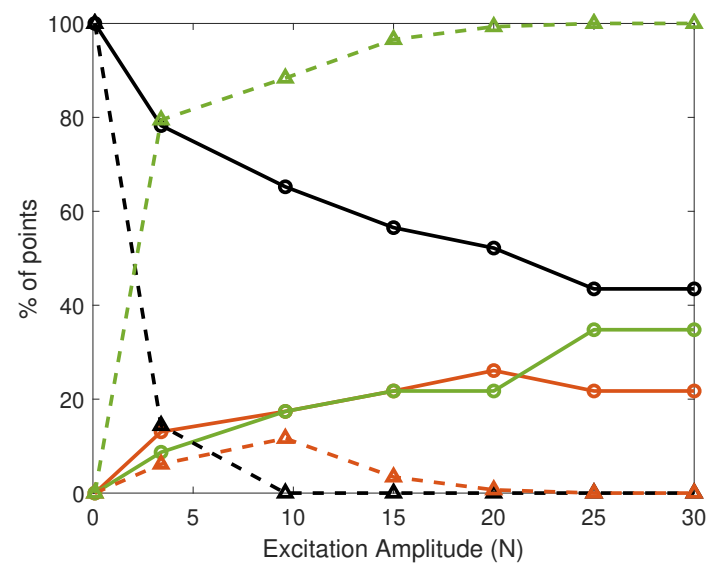

FIGURE 13: Evolution of the $\%$ of contact condition repartition at the resonance frequency for the conical (-) and the wedge $(\cdots .$.$) for different excitation amplitude - Full contact (black) -$ Stick/slip (orange) - Contact separation (green)

in the in-phase case is of the same order of magnitude than the energy dissipated by the conical damper. Hence, the conical and the wedge dampers have similar performances in terms of energy dissipation. However, the results obtained here for the wedge damper are based on the strong assumption that the contact surface is in perfect contact. In reality, the location and the surface area of the contact are unknown, and it results in a drop of the resonance frequency for this mode. Hence, the modelling of this damper is limited, and the resonance frequency cannot be estimated. On the opposite, the contact condition between the conical damper and the platforms is much more robust since it corresponds to a contact line that can be easily identified. It results in a strong ability to model and capture the behaviour of the structure. Moreover, the coupling between the platforms can be controlled, and so the frequency shift caused by the damper. For these different reasons, the conical damper appears more efficient and reliable than the wedge damper.

\section{CONCLUSION}

In the present study, a new damper geometry has been evaluated. Based on a conical geometry, the problem of pure rolling motion when the blades have an in-phase motion cannot occurs. Indeed, to maintain contact with the two platforms, slipping motion must takes place, and so friction appears. This friction is due to the pure kinematic motion of the platforms, and so the conical damper has a very high damping potential.

First, from geometrical considerations and some assumptions, an analytical formulation of the expected kinematic slip is determined. It allows a fast estimation of the performances of a damper for different geometric properties in a preliminary design process. Then, the real damping efficiency of the damper 
TABLE 1: Energy dissipated by friction in $\mathrm{mJ}$ for the different dampers and the different excitation levels for the out-of-phase mode

\begin{tabular}{llllllll}
\hline Force $(\mathbf{N})$ & $\mathbf{0 . 0 9 6}$ & $\mathbf{3 . 8 4}$ & $\mathbf{9 . 6}$ & $\mathbf{1 5}$ & $\mathbf{2 0}$ & $\mathbf{2 5}$ & $\mathbf{3 0}$ \\
\hline Wedge & $2.3 \mathrm{e}-22$ & $1.4 \mathrm{e}-3$ & $1.2 \mathrm{e}-2$ & $2.6 \mathrm{e}-2$ & $3.9 \mathrm{e}-2$ & $5.2 \mathrm{e}-2$ & $6.6 \mathrm{e}-2$ \\
Cylindrical & $1.3 \mathrm{e}-21$ & $1.8 \mathrm{e}-18$ & $1.1 \mathrm{e}-2$ & $2.4 \mathrm{e}-2$ & $3.5 \mathrm{e}-2$ & $4.7 \mathrm{e}-2$ & $5.8 \mathrm{e}-2$ \\
Conical & $8.1 \mathrm{e}-22$ & $1.7 \mathrm{e}-18$ & $1.1 \mathrm{e}-2$ & $2.3 \mathrm{e}-2$ & $3.5 \mathrm{e}-2$ & $4.6 \mathrm{e}-2$ & $5.8 \mathrm{e}-2$ \\
\hline
\end{tabular}

TABLE 2: Energy dissipated by friction in $\mathrm{mJ}$ for the different dampers and the different excitation levels for the in-phase mode

\begin{tabular}{llllllll}
\hline Force $(\mathbf{N})$ & $\mathbf{0 . 0 9 6}$ & $\mathbf{3 . 8 4}$ & $\mathbf{9 . 6}$ & $\mathbf{1 5}$ & $\mathbf{2 0}$ & $\mathbf{2 5}$ & $\mathbf{3 0}$ \\
\hline Wedge & $4.7 \mathrm{e}-21$ & $3.6 \mathrm{e}-3$ & $3.3 \mathrm{e}-2$ & $8.1 \mathrm{e}-2$ & $1.4 \mathrm{e}-1$ & $2.3 \mathrm{e}-1$ & $3.3 \mathrm{e}-1$ \\
Cylindrical & $6.2 \mathrm{e}-23$ & $1.7 \mathrm{e}-19$ & $1.6 \mathrm{e}-18$ & $1.0 \mathrm{e}-18$ & $4.1 \mathrm{e}-18$ & $6.2 \mathrm{e}-18$ & $9.5 \mathrm{e}-18$ \\
Conical & $3.9 \mathrm{e}-20$ & $1.8 \mathrm{e}-2$ & $5.9 \mathrm{e}-2$ & $1.0 \mathrm{e}-1$ & $1.4 \mathrm{e}-1$ & $1.8 \mathrm{e}-1$ & $2.2 \mathrm{e}-1$ \\
\hline
\end{tabular}

is determined by using a nonlinear dynamic analysis based on the HBM. The nonlinear receptances of the conical damper for different excitation levels are compared to the receptances of a cylindrical and a wedge damper. The damping properties of the different dampers are similar in the case of an out-of-phase motion of the blades. But in the case of an in-phase motion of the blades, the behaviours of the three dampers tend to be very different. The cylindrical damper has a pure linear behaviour and has almost no damping properties. The wedge damper has a strong softening behaviour, and so a non-negligible frequency shift. On the opposite, the conical damper has a high level of damping as it was expected from the analytical results. The energy dissipated by friction for the different dampers are almost compared and confirmed the pure rolling motion of the cylindrical damper in in-phase case. The energies dissipated by the wedge and the conical damper are of the same order of magnitude.

As a conclusion, the conical damper has a high damping potential for both in-phase and out-of-phase motion. The contact conditions are more robust than for the wedge damper since the contact corresponds to a contact line that can be easily located. Moreover, by increasing the cone angle, the coupling effect between the two platforms is increased, therefore, the frequency shift caused by the damper can be controlled by a simple controllable parameter. Furthermore, from simple design parameters (as the cone angle), a very fine trade-off between the damping efficiency and the wear rate can be achieved.

\section{ACKNOWLEDGEMENT}

The third and fourth authors are grateful to Innovate UK (Grant no. MEDY.P50254) and Rolls-Royce plc (Grant no. MEDY.P42978) for providing the financial support for this work and for giving permission to publish it. This work was part of a collaborative R\&T project SILOET II P19.6 which is co-funded by Innovate UK and Rolls-Royce plc. The first and third authors thank Rolls-Royce plc and the EPSRC for the support under the Prosperity Partnership Grant "Cornerstone: Mechanical Engineering Science to Enable Aero Propulsion Futures", Grant Ref: EP/R004951/1.

\section{REFERENCES}

[1] Krack, M., Salles, L., and Thouverez, F., 2017. "Vibration prediction of bladed disks coupled by friction joints". Archives of Computational Methods in Engineering, 24(3), pp. 589-636.

[2] Petrov, E., and Ewins, D., 2004. "State-of-the-art dynamic analysis for non-linear gas turbine structures". Proceedings of the Institution of Mechanical Engineers, Part G: Journal of Aerospace Engineering, 218(3), pp. 199-211.

[3] Cowles, B., 1996. "High cycle fatigue in aircraft gas turbines-an industry perspective". International Journal of Fracture, 80(2-3), pp. 147-163.

[4] Gaul, L., and Nitsche, R., 2001. "The role of friction in mechanical joints". Applied Mechanics Reviews, 54(2), pp. 93-106.

[5] Feeny, B., Guran, A., Hinrichs, N., and Popp, K., 1998. "A historical review on dry friction and stick-slip phenomena". Applied Mechanics Reviews, 51(5), pp. 321-341.

[6] Griffin, J., 1990. "A review of friction damping of turbine blade vibration". International Journal of Turbo and Jet Engines, 7(3-4), pp. 297-308.

[7] Szwedowicz, J., Gibert, C., Sommer, T., and Kellerer, R., 2008. "Numerical and experimental damping assessment 
of a thin-walled friction damper in the rotating setup with high pressure turbine blades". Journal of Engineering for Gas Turbines and Power, 130(1), p. 012502.

[8] Sanliturk, K., Ewins, D., and Stanbridge, A., 2001. "Underplatform dampers for turbine blades: theoretical modeling, analysis, and comparison with experimental data". Journal of Engineering for Gas Turbines and Power, 123(4), pp. 919-929.

[9] Sanliturk, K., Ewins, D., Elliott, R., and Green, J., 2001. "Friction damper optimization: simulation of rainbow tests". Journal of Engineering for Gas Turbines and Power, 123(4), pp. 930-939.

[10] Panning, L., Popp, K., Sextro, W., Kayser, A., and Wolter, I., 2004. "Asymmetrical underplatform dampers in gas turbine bladings: theory and application". In ASME Turbo Expo 2004: Power for Land, Sea, and Air, pp. 269-280.

[11] Petrov, E., and Ewins, D., 2007. "Advanced modeling of underplatform friction dampers for analysis of bladed disk vibration". Journal of Turbomachinery, 129(1), pp. 143150.

[12] Panning, L., Sextro, W., and Popp, K. "Optimization of interblade friction damper design". In ASME Turbo Expo 2000: Power for Land, Sea, and Air.

[13] Jareland, M., 2001. "A parametric study of a cottageroof damper and comparison with experimental results". In ASME Turbo Expo 2001: Power for Land, Sea, and Air.

[14] Firrone, C., Zucca, S., and Gola, M., 2009. "Effect of static/dynamic coupling on the forced response of turbine bladed disks with underplatform dampers". In ASME Turbo Expo 2009: Power for Land, Sea, and Air, pp. 429440.

[15] Pesaresi, L., Salles, L., Jones, A., Green, J., and Schwingshackl, C., 2017. "Modelling the nonlinear behaviour of an underplatform damper test rig for turbine applications". Mechanical Systems and Signal Processing, 85, pp. 662679.

[16] Jareland, M., 2001. "Experimental investigation of a platform damper with curved contact areas". In ASME Design Engineering Conference, Pittsburgh.

[17] Csaba, G., 1999. "Modelling of a microslip friction damper subjected to translation and rotation". In ASME 1999 international gas turbine and aeroengine congress and exhibition.

[18] Bessone, A., Toso, F., and Berruti, T., 2015. "Investigation on the dynamic response of blades with asymmetric under platform dampers". In ASME Turbo Expo 2015: Turbine Technical Conference and Exposition.

[19] Gola, M., and Gastaldi, C., 2014. "Understanding complexities in underplatform damper mechanics". In ASME Turbo Expo 2014: Turbine Technical Conference and Exposition.

[20] Zucca, S., Botto, D., and Gola, M., 2008. "Range of variability in the dynamics of semi-cylindrical friction dampers for turbine blades". In ASME Turbo Expo 2008: Power for Land, Sea, and Air, pp. 519-529.

[21] Gastaldi, C., and Gola, M., 2017. "Pre-optimization of asymmetrical underplatform dampers". Journal of Engineering for Gas Turbines and Power, 139(1), p. 012504.

[22] Pesaresi, L., Armand, J., Schwingshackl, C., Salles, L., and Wong, C., 2018. "An advanced underplatform damper modelling approach based on a microslip contact model". Journal of Sound and Vibration, 436, pp. 327-340.

[23] Petrov, E., and Ewins, D., 2003. "Analytical formulation of friction interface elements for analysis of nonlinear multiharmonic vibrations of bladed disks". Journal of turbomachinery, 125(2), pp. 364-371.

[24] Petrov, E., 2008. "Explicit finite element models of friction dampers in forced response analysis of bladed disks". Journal of engineering for gas turbines and power, 130(2), p. 022502.

[25] Petrov, E., 2011. "A high-accuracy model reduction for analysis of nonlinear vibrations in structures with contact interfaces". Journal of Engineering for Gas Turbines and Power, 133(10), p. 102503.

[26] Cameron, R., and Martin, W., 1947. "The orthogonal development of non-linear functionals in series of fourierhermite functionals". Annals of Mathematics, pp. 385-392.

[27] Salles, L., Blanc, L., Thouverez, F., Gouskov, A., and Jean, P., 2009. "Dynamic analysis of a bladed disk with friction and fretting-wear in blade attachments". In ASME Turbo Expo 2009: Power for Land, Sea, and Air, pp. 465-476.

[28] Ning, X., and Lovell, M., 2002. "On the sliding friction characteristics of unidirectional continuous frp deposits". ASME Journal of Tribology, 48(5), pp. 2000-2008. 